\title{
A equação escalar conservativa: aplicações em fluxo de tráfego
}

\section{The conservative scalar equation: traffic flow applications}

\author{
Faculdade de Formação de Professores, Universidade do Estado do Rio de Janeiro (FFP-UERJ), \\ Departamento de Matemática, São Gonçalo, RJ, Brasil \\ https://orcid.org/0000-0002-1015-6311, icaraujo@iprj.uerj.br
}

Rosa García Márquez Faculdade de Formação de Professores, Universidade do Estado do Rio de Janeiro (FFP-UERJ), Departamento de Matemática, São Gonçalo, RJ, Brasil https://orcid.org/0000-0003-3465-569X, rosagmarquez@yahoo.com.br

Millena Ribeiro Fontinha

Faculdade de Formação de Professores, Universidade do Estado do Rio de Janeiro (FFP-UERJ), Licenciatura em Matemática, São Gonçalo, RJ, Brasil https://orcid.org/0000-0001-8053-4739, millenaribeiro@yahoo.com.br

\section{Informações do Artigo \\ Como citar este artigo \\ ARAÚJO, Jorge Corrêa de; MÁRQUEZ, Rosa García; FONTINHA, Millena Ribeiro. A \\ equação escalar conservativa: aplicações em fluxo de tráfego. REMAT: Revista Eletrônica da Matemática, Bento Gonçalves, RS, v. 6 n. 2, p. e4005, 30 set. 2020. DOI: https://doi.org/10.35819/remat2020v6i2id4162}

Histórico do Artigo

Submissão: 27 de abril de 2020

Aceite: 22 de junho de 2020 .

\section{Palavras-chave}

Fluxo de Tráfego

Regularização de Dados Iniciais

Solução Fraca

\section{Resumo}

Neste trabalho, a equação escalar conservativa é utilizada para a representação de um modelo de fluxo de tráfego veicular com a abertura de sinal após a luz verde em uma rodovia com dois diferentes campos de velocidades em função da densidade de veículos. Duas metodologias foram apresentadas, uma delas é derivada de uma equação algébrica e a outra, denominada regularização dos dados iniciais, consiste em tomar o limite das aproximações contínuas das condições iniciais $u^{\varepsilon}(x, 0)$ para obter a solução do problema, o que constitui a originalidade de nossa contribuição. A simulação realizada com os modelos adotados neste estudo mostrou que o fluxo de veículos durante o sinal aberto é mais intenso com o modelo quadrático.
Keywords

Traffic Flow

Regularization of Initial Data

Weak Solution

\begin{abstract}
In this work, the conservative scalar equation was used to represent a vehicular traffic flow model with the signal opening after the green light on a highway with two different speed fields as a function of car density. Two methodologies were presented, one of them is derived from an algebraic equation and the other, called initial regularization data, consists of taking the limit of the continuous approximations of the initial conditions $u^{\varepsilon}(x, 0)$ to obtain the problem solution, what constitutes the originality of this article. The simulation performed with the adopted models in this study, showed that the flow of vehicles during the open signal is more intense with the quadratic model.
\end{abstract}




\section{Introdução}

Uma equação escalar conservativa na forma diferencial é dada por (LEVEQUE, 1992)

$$
u_{t}+(f(u))_{x}=0,
$$

onde $f(u)$ é uma função diferenciável.

Tais equações são equações diferenciais parciais do tipo hiperbólico, nas quais segundo Cuminato e Meneguette Junior (2013), as descontinuidades são transportadas sem suavização, podendo haver a formação de singularidades ou choques, mesmo no caso de dados iniciais bem comportados. A equação (1) com a condição inicial dada na equação (2) pela função Heaviside constitui um problema de Cauchy, também conhecido como problema de valor inicial ou problema de Riemann (LEVEQUE, 1992),

$$
H(x)=\left\{\begin{array}{ll}
u_{e}, & x \leq 0 \\
u_{d}, & x>0
\end{array},\right.
$$

no qual $u_{e}$ e $u_{d}$ são constantes. Tais problemas em geral não possuem soluções clássicas ou globais, ou seja, soluções que sejam contínuas e diferenciáveis. Entretanto, é possível a admissão de soluções fracas, no sentido de soluções clássicas por partes (CUMINATO; MENEGUETTE JUNIOR, 2013), isto é, uma solução seccionalmente contínua e diferenciável. Uma definição rigorosa de solução fraca pode ser encontrada em Leveque (1992).

De acordo com Cuminato e Meneguette Junior (2013), quando soluções fracas são admitidas, o problema de valor inicial pode admitir uma multiplicidade de soluções. Um dos critérios para a escolha da solução fraca mais adequada para o problema analisado, denominada solução entrópica (CUMINATO; MENEGUETTE JUNIOR, 2013), é o da regularização dos dados iniciais, que consiste em tomar o limite das soluções de uma sequência de problemas com condições iniciais $u^{\varepsilon}(x, 0)$ contínuas, convergindo pontualmente para a condição inicial do problema original (CUMINATO; MENEGUETTE JUNIOR, 2013). Dito de outro modo, a solução entrópica pode ser obtida tomando o limite das aproximações contínuas das condições iniciais $u^{\varepsilon}(x, 0)$.

Algumas aplicações práticas da equação escalar conservativa envolvem, por exemplo, modelos de fluxo de tráfego e recuperação de petróleo. Um modelo de fluxo de tráfego em uma rodovia após a abertura do sinal com luz verde foi proposto por Haberman (1977). Nesse modelo, o campo de velocidade foi relacionado de modo linear com a densidade de veículos antes e após a abertura do sinal depois de decorrido um tempo de longa duração do sinal fechado. Haberman não usou a regularização dos dados iniciais, em vez dessa técnica, Haberman (1977) obteve a função de densidade de carros como função da posição e do tempo por meio da solução de uma equação algébrica envolvendo a taxa de fluxo de carros e as retas características partindo da origem, onde é construída a solução do problema de Riemann na região de rarefação. Nesse 
trabalho, o modelo de tráfego, anteriormente descrito, foi resolvido de forma alternativa usando a regularização de dados iniciais, pois a metodologia utilizada por Haberman (1977), pode resultar em equações algébricas impossíveis de serem resolvidas em função da densidade. Portanto, em vez de equações algébricas, o problema proposto foi resolvido via parametrização $x_{0}=x_{0}(x, t)$ dos dados iniciais para os modelos de campo linear e quadrático. Mesmo a regularização de dados iniciais, pode esbarrar na dificuldade de obter a parametrização da posição inicial na rarefação, $x_{0}=x_{0}(x, t)$ necessária nesse tipo de procedimento. Essa dificuldade na regularização é exemplificada neste trabalho com o uso de um modelo quadrático para o campo de velocidade relacionado à densidade de fluxo.

\section{Materiais e métodos}

Nesta seção, apresentaremos a equação escalar conservativa e o método das características.

\subsection{A equação escalar conservativa}

A lei da conservação de massa, caso homogêneo, é expressa matematicamente na forma integral como (FOX; PRITCHARD; MCDONALD, 2015)

$$
\frac{\partial}{\partial t} \int_{\Omega} \rho d V+\int_{\partial \Omega} \rho \vec{V} \vec{d} \sigma=0,
$$

sendo $\vec{d} \sigma=\vec{\eta} \cdot d \sigma$, onde $\vec{\eta}$ é o vetor normal ao elemento de área $d \sigma$ da superfície $\partial \Omega$ da região $\Omega$, denominada volume de controle $(\mathrm{VC})$ situada no espaço tridimensional onde o fluído escoa. O primeiro termo integral da equação (3) é a taxa de variação de massa $\frac{d M}{d t}$ de uma substância de concentração $\rho=\rho(\vec{x}, t)$ dissolvida no fluído com campo de velocidade $\vec{V}=\vec{V}(\vec{x}, t)$. O produto $\rho \vec{V}$ é a densidade de fluxo de massa, isto é, a quantidade de substância que atravessa uma unidade de área da superfície de controle por unidade de tempo. Se $\frac{\partial \rho}{\partial t}$ é contínua em $\Omega$, tem-se segundo Williamson, Crowell e Trotter (1979) que

$$
\frac{d M}{d t}=\int_{\Omega} \frac{\partial \rho}{\partial t} d V
$$

Das equações (3) e (4), do teorema da divergência (GUIDORIZZI, 2019) e da linearidade das integrais triplas tem-se

$$
\int_{\Omega}\left[\frac{\partial \rho}{\partial t}+\nabla \cdot \rho \vec{V}\right] d V=0
$$


Como a equação (5) é válida para qualquer VC arbitrário, resulta a forma diferencial da equação de conservação de massa dada por

$$
\frac{\partial \rho}{\partial t}+\nabla \cdot \rho \vec{V}=0
$$

No caso unidimensional, a equação (6) fica na forma escalar dada pela equação diferencial parcial hiperbólica

$$
\frac{\partial \rho}{\partial t}+\frac{\partial(\rho V)}{\partial x}=0
$$

Definindo $f(\rho)=\rho V$ como uma função de fluxo, a equação (7) fica na forma conservativa dada por

$$
\frac{\partial \rho}{\partial t}+\frac{\partial f(\rho)}{\partial x}=0
$$

ou segundo Leveque (1992) como

$$
\rho_{t}+(f(\rho))_{x}=0 .
$$

Em particular, se $\vec{V}=v \vec{i}$, onde $v$ é uma constante e $\vec{i}$ é o vetor canônico, a equação (8) pode ser escrita na forma

$$
\frac{\partial \rho}{\partial t}+v \frac{\partial \rho}{\partial x}=0
$$

A equação (10) é denominada equação linear de advecção (LEVEQUE, 1992) ou equação de transporte de massa.

\subsection{O método das características}

O objetivo desse método é encontrar curvas $x=x(t)$ onde $\rho(x(t), t)$ seja constante ao longo dessas curvas para a construção da solução clássica por partes (solução fraca) do problema de Riemann. Da equação (9) e usando a regra da cadeia, obtemos

$$
\rho_{t}+f^{\prime}(\rho) \rho_{x}=0 \text {. }
$$

Como $\rho=\rho(x, t)$ usando a diferencial total $\frac{d \rho}{d t}$, obtemos

$$
\frac{d \rho}{d t}=\frac{\partial \rho}{\partial t}+\frac{\partial \rho}{\partial x} \frac{d x}{d t}
$$

Das equações (11) e (12) segue que $\frac{d \rho}{d t}=0$, ou seja, $\rho$ é constante no tempo se

$$
\frac{d x}{d t}=f^{\prime}(\rho)=\frac{d f(\rho)}{d \rho}
$$


A equação (13) é uma equação diferencial ordinária de variáveis separáveis. Considerando $x_{0}=x(0)$ um ponto genérico do eixo $x$, a solução da equação (13) são retas características na forma

$$
x=x_{0}+\left(\frac{d f(\rho)}{d \rho}\right) t .
$$

Portanto, ao longo de cada uma das características dadas pela equação (14) tem-se

$$
\rho(x(t), t)=\rho(x(0), 0)=\rho\left(x_{0}, 0\right)=\rho\left(x_{0}\right)=\rho_{0} .
$$

Ou seja, a solução ao longo de cada característica depende somente da solução ou condição inicial. No caso da equação (10), a solução seria formada por retas características paralelas no plano $x t$, na qual a velocidade de propagação de cada reta é constante e igual a $f^{\prime}\left(\rho_{0}\right)=\frac{d f\left(\rho_{0}\right)}{d \rho}$. A equação (14) pode fornecer de modo direto características para uma região do plano $x t$ partindo de $x_{0}$, e da solução algébrica da equação (14) obter a expressão para $\rho=\rho(x, t)$ nessa mesma região, destaca-se que essa foi a metodologia adotada por Haberman (1977) para um problema de fluxo de tráfego.

\section{Aplicação em fluxo de tráfego: rodovia com o início da luz verde}

Seja $u=u(x, t)$ um campo de velocidade em uma rodovia de faixa única sem entradas ou saídas laterais, isto é, o número de carros é conservado. Esse campo estima a velocidade de um carro, medido por um observador situado na posição $x$ no tempo $t$. Se $x_{i}(t)$ é a posição individual de um carro nessa rodovia, sua velocidade é dada pela cinemática elementar como $u_{i}(t)=\frac{d x_{i}}{d t}(t)$. Segundo Haberman (1977), pode ser estabelecida uma relação de igualdade entre essas velocidades como

$$
u\left(x_{i}(t), t\right)=u_{i}(t)=\frac{d x_{i}}{d t}(t) .
$$

Por fluxo de tráfego, $f=f(x, t)$ será considerado o número de carros passando pela posição $x$ da rodovia em um determinado tempo fixado, enquanto a densidade de tráfego, $\rho=\rho(x, t)$ é uma função da posição em um tempo fixado, por exemplo, a quantidade de carros em um km na rodovia em um determinado tempo fixado. Portanto, o fluxo deve ser igual ao produto da densidade pela velocidade, isto é, caso as variáveis que representam o tráfego dependam de $x$ e $t$ tem-se, segundo Haberman (1977)

$$
f(x, t)=\rho(x, t) u(x, t) .
$$

Com essas considerações a equação (7) reescreve-se na forma 


$$
\frac{\partial \rho}{\partial t}+\frac{\partial(\rho u)}{\partial x}=0
$$

Se o campo $u$ é conhecido, a equação (18) é uma equação diferencial parcial na função incógnita da função é a densidade de tráfego e sua solução. Uma vez conhecida a condição inicial, ela pode ser usada para obter a densidade de tráfego em uma rodovia. Das equações (16) e (18) tem-se

$$
\frac{\partial \rho}{\partial t}+\frac{\partial f}{\partial x}=0
$$

A equação (18) é chamada equação de "conservação de carros" (HABERMAN, 1977). Vamos admitir que $u=u(\rho)$, isto é, o campo de velocidade é função da densidade de tráfego. A partir dela e da equação (17), podemos escrever

$$
f(\rho)=\rho \cdot u(\rho) .
$$

Vamos assumir algumas hipóteses simplificadoras naturais considerando um tráfego em rodovia com as condições de conservação de carros, com o início de luz verde ou a abertura do sinal fechado depois de um tempo de longa duração:

1. $u=u(\rho)$, esta condição indica que o campo de velocidade é função da densidade de tráfego;

2. $u(0)=u_{\max }$, isto é, a velocidade é máxima quando a densidade é nula;

3. $\frac{d u}{d \rho} \leq 0$, expressa o fato de que a velocidade é decrescente em função da densidade;

4. $u\left(\rho_{\max }\right)=0$, indica que a velocidade é nula quando a densidade é máxima.

Tais condições, propostas por Haberman (1977), servem de base para a definição de campos de velocidades antes e depois da abertura do sinal. No tempo $t=0$, o sinal fica com luz verde em $x=0$. O problema de Riemann a ser resolvido é dado pela equação (11) com a condição inicial

$$
\begin{gathered}
\rho_{t}+\frac{d f}{d \rho} \frac{\partial \rho}{\partial x}=0, \\
\rho(x, 0)= \begin{cases}\rho_{\max }, & x<0 \\
0, & x>0 .\end{cases}
\end{gathered}
$$

Usando as equações (14), (15) e (20) têm-se as equações das retas características dadas por:

$$
x=\frac{d f(\rho)}{d \rho} t+x_{0}=\left(\rho \frac{d u(\rho)}{d \rho}+u(\rho)\right) t+x_{0}=\left(\rho_{0} \frac{d u\left(\rho_{0}\right)}{d \rho}+u\left(\rho_{0}\right)\right) t+x_{0}
$$


Para $x<0$, da equação (22) tem-se $\rho(x, 0)=\rho_{0}=\rho_{\max }$. Logo, da equação (23) e da condição (iv) as características nessa região com $x_{0}<0$ são dadas por

$$
x=x_{0}+\rho_{\max }\left(\frac{d u\left(\rho_{\max }\right)}{d \rho}\right) t .
$$

Para $x>0$, da equação (22) tem-se $\rho(x, 0)=\rho_{0}=0$. Logo, da equação (23) e da condição (ii) as características nessa região com $x_{0}>0$ são dadas por

$$
x=x_{0}+u_{\max } t
$$

Da condição (iii) tem-se $\frac{d u(\rho)}{d \rho} \leq 0$, a fronteira da região no plano $x t$ de densidade máxima é limitada à direita pela característica $x=\rho_{\max }\left(\frac{d u\left(\rho_{\max }\right)}{d \rho}\right) t$ que parte da posição $x_{0}=0$ no tempo $t=0$, enquanto a fronteira da região de densidade nula é limitada à esquerda pela característica $x=u_{\max } t$ que parte da posição $x_{0}=0$ no tempo $t=0$. Portanto, existe uma região ausente de retas características, denominada de região de rarefação dada por

$$
\rho_{\max }\left(\frac{d u\left(\rho_{\max }\right)}{d \rho}\right) t<x<u_{\max } t .
$$

\subsection{Campo de velocidade linear}

O problema de início de movimentação de carros após a luz verde foi analisado por Haberman (1977) considerando o campo de velocidade linear definido por

$$
u(\rho)=u_{\max }\left(1-\frac{\rho}{\rho_{\max }}\right), \quad 0<\rho \leq \rho_{\max },
$$

onde as características na rarefação foram obtidas diretamente da equação (14) com $x_{0}=0$, o que permite uma solução algébrica da equação (13) para a determinação da densidade de tráfego nessa região, completando a solução fraca para o problema de Riemann. Entretanto, a justificativa apresentada por Haberman (1977) para as características na rarefação foi puramente formal, ou seja, foram usados argumentos intuitivos de limites de funções.

Haberman (1977) argumentou que as características partindo da descontinuidade seriam obtidas como o limite das características $x=x_{0}+\left(\frac{d f(\rho)}{d \rho}\right) t$ quando $\Delta x \rightarrow 0$, o que de fato é intuitivo. Dessa maneira, Haberman usou a equação (14) com $x_{0}=0$ e as equações (20) e (27) para obter a solução algébrica da densidade de tráfego na rarefação dada por 


$$
\rho=\frac{\rho_{\max }}{2}\left(1-\frac{x}{u_{\max } t}\right), \quad-u_{\max } t<x<u_{\max } t .
$$

Em oposição a essa metodologia descrita anteriormente, vamos obter a solução fraca desse problema de Riemann usando o limite de uma sequência de problemas com condições iniciais contínuas $u^{\varepsilon}(x, 0)$ convergindo pontualmente para a condição inicial original (CUMINATO; MENEGUETTE JUNIOR, 2013). A Figura 1 mostra a região das características e a rarefação com o campo de velocidade linear dado pela equação (27).

Figura 1 - Características e rarefação no plano $x t$ para diferentes valores de $x_{0}$.

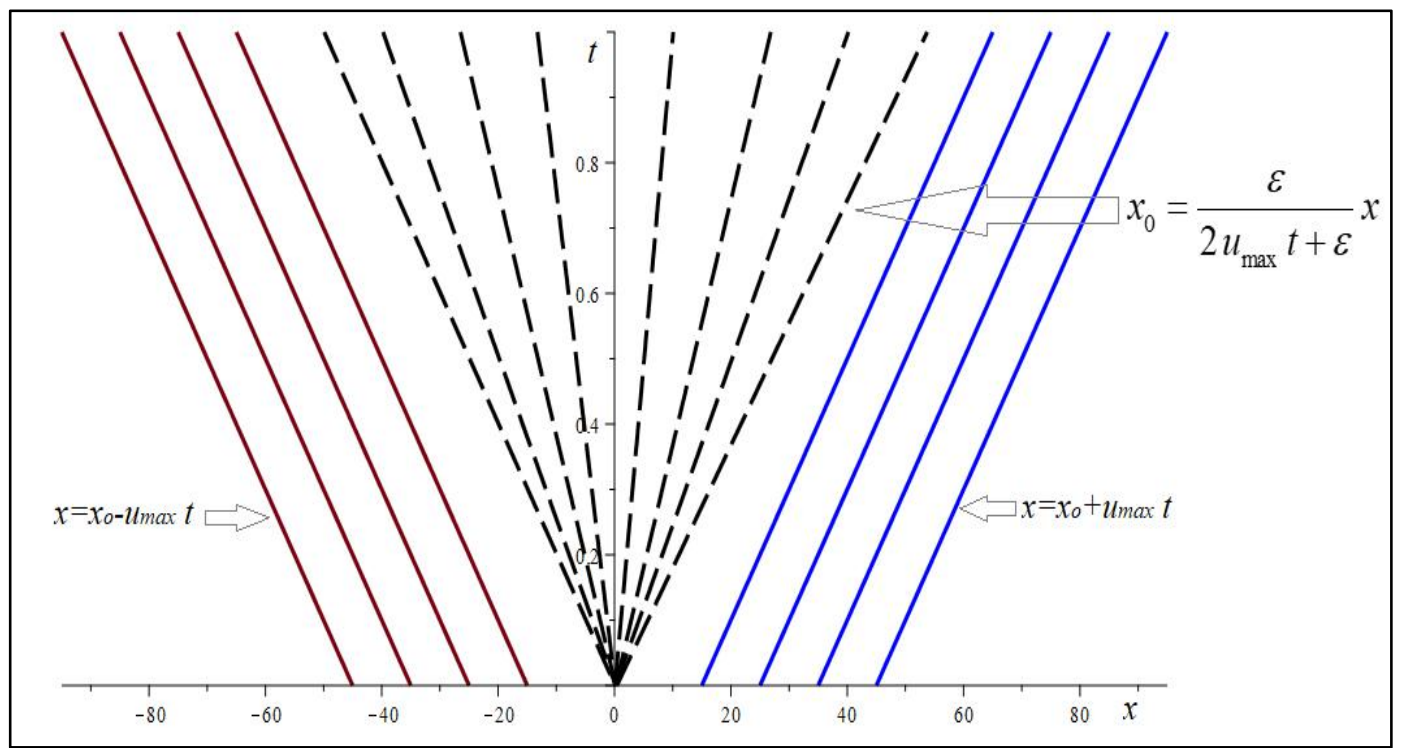

Fonte: Elaboração dos autores (2020).

Pode-se regularizar a descontinuidade na origem dada pela equação (22) por meio de sequências de condições iniciais contínuas, $\rho^{\varepsilon}(x, 0)$, com $\varepsilon>0$ definidas por

$$
\rho^{\varepsilon}(x, 0)=\left\{\begin{array}{lc}
\rho_{\max }, & x \leq-\frac{\varepsilon}{2}, \\
\underbrace{\frac{\rho_{\max }}{2}-\frac{\rho_{\max }}{\varepsilon} x}_{L(x)}, & -\frac{\varepsilon}{2}<x<\frac{\varepsilon}{2}, \\
0, & x \geq \frac{\varepsilon}{2},
\end{array}\right.
$$

onde $L(x)$ é uma bijeção linear decrescente ligando os pares de pontos $\left(\frac{-\varepsilon}{2}, \rho_{\max }\right)$ e $\left(\frac{\varepsilon}{2}, 0\right)$. Das equações (20) e (27) tem-se

$$
\frac{d f}{d \rho}=u_{\max }\left(1-\frac{2 \rho}{\rho_{\max }}\right) .
$$

Usando as equações (15), (29) e (30) obtemos 


$$
\frac{d f\left(\rho^{\varepsilon}(x, t)\right)}{d \rho}=\frac{d f\left(\rho^{\varepsilon}(x, 0)\right)}{d \rho}=\frac{d f\left(L\left(x_{0}\right)\right)}{d \rho}=\underbrace{\frac{2 u_{\max }}{\varepsilon} x_{0}}_{\alpha}, \quad-\frac{\varepsilon}{2}<x<\frac{\varepsilon}{2} .
$$

Das equações (14), (15) e (31) as características na rarefação, isto é, começando em $x_{0}$, $-\frac{\varepsilon}{2}<x_{0}<\frac{\varepsilon}{2}$ são dadas por

$$
x_{0}=\frac{\varepsilon}{2 u_{\max } t+\varepsilon} x .
$$

Note que $x_{0}=x_{0}(x, t)$, ou seja, $x_{0}$ é parametrizado em função das variáveis $x$ e $t$. É possível mostrar que as inclinações, $\alpha$ dadas pela equação (31) das características na rarefação variam continuamente no intervalo $-u_{\max }<\alpha<u_{\max }$. Como $\rho^{\varepsilon}(x, t)$ é constante ao longo de cada reta característica na rarefação, tem-se das equações (29) e (32) que

$$
\rho^{\varepsilon}(x, t)=\rho^{\varepsilon}\left(x_{0}, 0\right)=-\frac{\rho_{\max }}{\varepsilon}\left(\frac{\varepsilon}{2 u_{\max } t+\varepsilon}\right) x+\frac{\rho_{\max }}{2}=-\frac{\rho_{\max }}{2 u_{\max } t+\varepsilon} x+\frac{\rho_{\max }}{2} .
$$

Portanto, uma solução entrópica do problema de Riemann, dado pelas equações (21) e (29) é da forma

$$
\rho^{\varepsilon}(x, t)=\left\{\begin{array}{lc}
\rho_{\text {max }}, & x \leq-\frac{\varepsilon}{2}-u_{\text {max }} t, \\
\frac{-\rho_{\max }}{2 u_{\max } t+\varepsilon} x+\frac{\rho_{\max }}{2}, & -u_{\max } t-\frac{\varepsilon}{2}<x<u_{\max } t+\frac{\varepsilon}{2} \\
0, & x \geq \frac{\varepsilon}{2}+u_{\max } t .
\end{array}\right.
$$

A solução entrópica do problema de Riemann, dado pelas equações (21) e (22) é

$$
\rho(x, t)=\lim _{\varepsilon \rightarrow 0} \rho^{\varepsilon}(x, t)=\left\{\begin{array}{cc}
\rho_{\max }, & x \leq-u_{\max } t, \\
\frac{\rho_{\max }}{2}\left(1-\frac{x}{u_{\max } t}\right), & -u_{\max } t<x<u_{\max } t, \\
0, & x \geq u_{\max } t,
\end{array}\right.
$$

onde a equação (35) na rarefação coincide com a equação (28) obtida por Haberman (1977).

\subsection{Campo de velocidade quadrática}

Vamos usar agora um campo de densidade de velocidade quadrática dado por

$$
u(\rho)=u_{\max }\left(1-\frac{\rho^{2}}{\rho_{\max }^{2}}\right), \quad 0 \leq \rho \leq \rho_{\max } .
$$

Da equação (36) tem-se

$$
\frac{d u}{d \rho}=-\frac{2 u_{\max }}{\rho_{\max }^{2}} \rho
$$


Considerando os dados anteriores e a equação (20) tem-se

$$
\frac{d f}{d \rho}=u_{\max }\left(1-\frac{3 \rho^{2}}{\rho_{\max }^{2}}\right) \text {. }
$$

O campo de velocidade quadrático dado pela equação (36) satisfaz as condições simplificadoras aqui adotadas para a lei de conservação de tráfego em rodovia com o início da movimentação de veículos, após a abertura de sinal em $x=0$ no tempo $t=0$. Das equações (23) e (37) obtêm-se as equações características à esquerda e a direita de $x_{0}$ dadas por

$$
\begin{gathered}
x=\rho \max \frac{d u(\rho \max )}{d \rho} t-x_{0}=-2 u \text { max } t+x_{0}, x_{0}<0 \\
x=u \text { max } t+x_{0}, x_{0}>0
\end{gathered}
$$

Para calcular a densidade na região de rarefação $-2 u_{\max } t<x<u_{\max } t$, para $t>0$, pode-se usar o procedimento adotado por Haberman (1977), o qual utiliza as equações (13) e (38), conforme já fora discutido, para obter a equação algébrica

$$
u_{\max }\left(1-\frac{3 \rho^{2}}{\rho_{\max }^{2}}\right)=\frac{x}{t} .
$$

A equação (41) pode ser posta na forma explícita, $\rho=\rho(x, t)$ como

$$
\rho=\frac{1}{\sqrt{3}} \rho_{\max }\left(1-\frac{x}{u_{\max } t}\right)^{1 / 2} .
$$

A solução fraca do problema de Riemann, dado pelas equações (21) e (22) com o campo de densidade de velocidade quadrática é então dada por

$$
\rho(x, t)=\left\{\begin{array}{cc}
\rho_{\text {max }}, & x<-2 u_{\text {max }} t, \\
\frac{\sqrt{3} \rho_{\text {max }}}{3} \sqrt{1-\frac{x}{u_{\text {max }} t},} & -2 u_{\max } t \leq x \leq u_{\text {max }} t, \\
0, & x>u_{\text {max }} t .
\end{array}\right.
$$

A obtenção de $\rho=\rho(x, t)$ pela regularização dos dados iniciais, se dá utilizando as equações (31), (38) e a reta $L(x)=\frac{\rho_{\max }}{2}-\frac{\rho_{\max }}{\varepsilon} x$, definida no intervalo $-\frac{\varepsilon}{2}<x<\frac{\varepsilon}{2}$, onde

$$
\frac{d f\left(\rho^{\varepsilon}(x, t)\right)}{d \rho}=\frac{d f\left(\rho^{\varepsilon}(x, 0)\right)}{d \rho}=\frac{d f\left(L\left(x_{0}\right)\right)}{d \rho}=\frac{d f\left(\frac{\rho_{\max }}{2}-\frac{\rho_{\max } x_{0}}{\varepsilon}\right)}{d \rho} \text {. }
$$

Usando a equação (38) tem-se

$$
\frac{d f\left(\rho^{\varepsilon}(x, t)\right)}{d \rho}=u_{\max }\left[1-3\left(\frac{x_{0}}{\varepsilon}-\frac{1}{2}\right)^{2}\right], \quad-\frac{\varepsilon}{2}<x<\frac{\varepsilon}{2} .
$$


Das equações (14) e (44) têm-se as características na rarefação dadas por

$$
x=x_{0}+\frac{d f\left(L\left(x_{0}\right)\right)}{d \rho}=x_{0}+u_{\max }\left[1-3\left(\frac{x_{0}}{\varepsilon}-\frac{1}{2}\right)^{2}\right] t .
$$

A ideia é obter a parametrização da equação (45) na forma $x_{0}=x_{0}(x, t)$, como na equação (32), pois sabemos que $\rho^{\varepsilon}(x, t)=\rho^{\varepsilon}\left(x_{0}, 0\right)$ sobre quaisquer pontos $(x, t)$ que façam parte da reta característica partindo de $x_{0}$. A equação (45) pode então ser colocada na forma

$$
\frac{x}{\varepsilon}=\frac{x_{0}}{\varepsilon}+\frac{u_{\text {max }} t}{\varepsilon}-3 \frac{u_{\text {max }} t}{\varepsilon}\left(\frac{x_{0}}{\varepsilon}-\frac{1}{2}\right)^{2} t,
$$

ou ainda como,

$$
3 \frac{u_{\max } t}{\varepsilon}\left(\frac{x_{0}}{\varepsilon}-\frac{1}{2}\right)^{2} t+\frac{x}{\varepsilon}-\frac{x_{0}}{\varepsilon}-\frac{u_{\max } t}{\varepsilon}=0
$$

Da equação (47) pode-se obter a equação quadrática em $\left(\frac{x_{0}}{\varepsilon}-\frac{1}{2}\right)$ dada por

$$
3 \frac{u_{\max } t}{\varepsilon}\left(\frac{x_{0}}{\varepsilon}-\frac{1}{2}\right)^{2}-\left(\frac{x_{0}}{\varepsilon}-\frac{1}{2}\right)+\left(\frac{x}{\varepsilon}-\frac{1}{2}\right)-\frac{u_{\max } t}{\varepsilon}=0 .
$$

Como $x_{0}<\frac{\varepsilon}{2}$, a raiz negativa deve ser escolhida para obter

$$
x_{0}=\frac{\varepsilon}{2}+\frac{\varepsilon^{2}}{6 u_{\max } t}+\frac{\varepsilon}{6 u_{\max } t} \sqrt{\varepsilon^{2}-12 u_{\max } x t+6 \varepsilon u_{\max } t+12 u_{\max }^{2} t^{2}} .
$$

Das equações (29) e (49) tem-se a densidade na rarefação $-2 u_{\max } t \leq x \leq u_{\max } t$, na forma

$$
\rho^{\varepsilon}(x, t)=\rho^{\varepsilon}\left(x_{0}, 0\right)=\frac{1}{6} \frac{\rho_{\max }}{u_{\max } t}\left[-\varepsilon+\sqrt{\varepsilon^{2}-12 u_{\max } x t+6 \varepsilon u_{\max } t+12 u_{\max }^{2} t^{2}}\right] .
$$

A solução do problema de Riemann, dado pelas equações (21) e (22), pode ser obtida da equação (50), na qual $x_{0}$ é dado na equação (49). Desse modo, a solução fraca pode ser reescrita como

$$
\rho(x, t)=\lim _{\varepsilon \rightarrow o} \rho^{\varepsilon}(x, t)=\left\{\begin{array}{lc}
\rho_{\text {max }}, & x \leq-2 u_{\text {max }} t \\
\frac{1}{6} \frac{\rho_{\text {max }}}{u_{\text {max }} t} \sqrt{12 u_{\text {max }}^{2} t^{2}-12 u_{\text {max }} x t}, & -2 u_{\text {max }} t<x<u_{\text {max }} t \\
0, & x \geq u_{\text {max }} t .
\end{array}\right.
$$

Observe que da equação (51) obtemos novamente a equação (43) na rarefação

$$
\begin{aligned}
\rho(x, t) & =\frac{1}{6} \frac{\rho_{\max }}{u_{\text {max }} t} \sqrt{12 u_{\text {max }}^{2} t^{2}-12 u_{\text {max }} x t}=\frac{\rho_{\max }}{6} \sqrt{12-\frac{12 x}{u_{\max } t}} \\
& =\frac{\sqrt{3} \rho_{\max }}{3} \sqrt{1-\frac{x}{u_{\max } t}},
\end{aligned}
$$

conforme o esperado. 
Na equação (24), a característica mais à esquerda da região de rarefação é dada por

$$
x=\rho_{\max }\left(\frac{d u\left(\rho_{\max }\right)}{d \rho}\right) t .
$$

Essa característica carrega a informação para trás, que o sinal abriu em $t=0$ com a velocidade constante $v=\rho_{\max }\left(\frac{d u\left(\rho_{\max }\right)}{d \rho}\right)$.

\section{Resultados e discussões}

No primeiro modelo analisado, na seção 3.1, ao utilizar as equações (24) e (27) obtém-se a equação da velocidade da característica à esquerda da região de descontinuidade, dada por

$$
v_{1}=-u_{\max },
$$

enquanto no segundo modelo, apresentado na seção 3.2, a velocidade da característica à esquerda da região de descontinuidade pode ser obtida da equação (39), na forma

$$
v_{2}=-2 u_{\max } .
$$

Logo, usando a cinemática do movimento uniforme, um motorista situado em $x_{0}<0$ com o modelo quadrático levará a metade do tempo para acelerar na rarefação em relação ao mesmo motorista com o modelo linear. É possível usar a equação (16) e os campos de velocidade obtidos pelas equações (27) e (36), bem como, as suas respectivas densidades na rarefação dadas pelas equações (28) e (42) para obter a equação da velocidade e da trajetória de um carro na rarefação com as representações adotadas. A obtenção desses parâmetros é oriunda de equações diferenciais ordinárias com coeficientes variáveis. Veremos com detalhes o modelo quadrático com base no modelo linear analisado por Haberman (1977).

No modelo linear, a característica $x=-u_{\max } t$ propaga com velocidade constante $-u_{\max }$ a informação da abertura do sinal em $t=0$ para a região de densidade máxima. Logo, um motorista situado em $x=-x_{0}<0$ levará um tempo $t=\frac{x_{0}}{u_{\max }}$ para começar a acelerar, enquanto que no modelo quadrático esse tempo é reduzido pela metade.

Das equações (16), (36) e (43) decorrido o tempo $t=\frac{x_{0}}{2 u_{\max }}$ obtemos o seguinte problema de Cauchy dado por

$$
\left\{\begin{array}{l}
t \frac{d x}{d t}-\frac{1}{3} x=\frac{2}{3} u_{\max } t, \quad t>0 \\
x=-x_{0}, \quad t=\frac{x_{0}}{2 u_{\max }} .
\end{array}\right.
$$


Como $t$ é não nulo, a equação (56) pode ser escrita como uma equação diferencial linear na forma

$$
\frac{d x}{d t}-\frac{x}{3 t}=\frac{2}{3} u_{\max }
$$

e solução geral da equação (57) que é dada por

$$
x(t)=c t^{1 / 3}+u_{\max } t .
$$

Considerando a condição inicial dada na equação (56), determinamos o valor da constante da equação (58). Assim, a solução do problema de valor inicial é dada por

$$
x=u_{\max } t-\frac{3}{2}\left(2 x_{0}^{2} u_{\max } t\right)^{\frac{1}{3}} .
$$

A equação (59) nos dá a posição particular de um carro na rarefação. É possível usar a equação (59) para obter o tempo necessário para que esse carro passe com a luz verde com o modelo quadrático. Para isso, basta resolver a equação (59) com $x=0$

$$
t=\frac{3 \sqrt{3} x_{0}}{2 u_{\max }} .
$$

A velocidade do carro pode ser obtida diretamente da equação (59) como

$$
\frac{d x}{d t}=u_{\max }-\frac{\sqrt[3]{2 x_{0}^{2} u_{\max } t}}{2 t}
$$

Note que quando $t \rightarrow \infty, v=\frac{d x}{d t} \rightarrow u_{\max }$, que é a velocidade máxima alcançada na região de densidade mínima imediatamente após a região de rarefação.

\subsection{Algumas simulações}

Nesta seção, realizaremos algumas simulações envolvendo o campo de velocidade quadrático dado pela equação (36). Para isto, vamos supor que em determinado sinal se observou que a densidade máxima por $\mathrm{km}$ é igual a $\rho_{\max }=250$ carros, e que a velocidade máxima permitida é de $u_{\max }=50(\mathrm{~km} / \mathrm{h})$. A função fluxo de veículos é dada pelas equações (20) e (36) como

$$
f(\rho)=\rho u_{\max }\left(1-\frac{\rho^{2}}{\rho_{\max }^{2}}\right) .
$$

A Figura 2 mostra o perfil do fluxo de tráfego em função da densidade de veículos. 
Figura 2 - Gráfico da função fluxo.

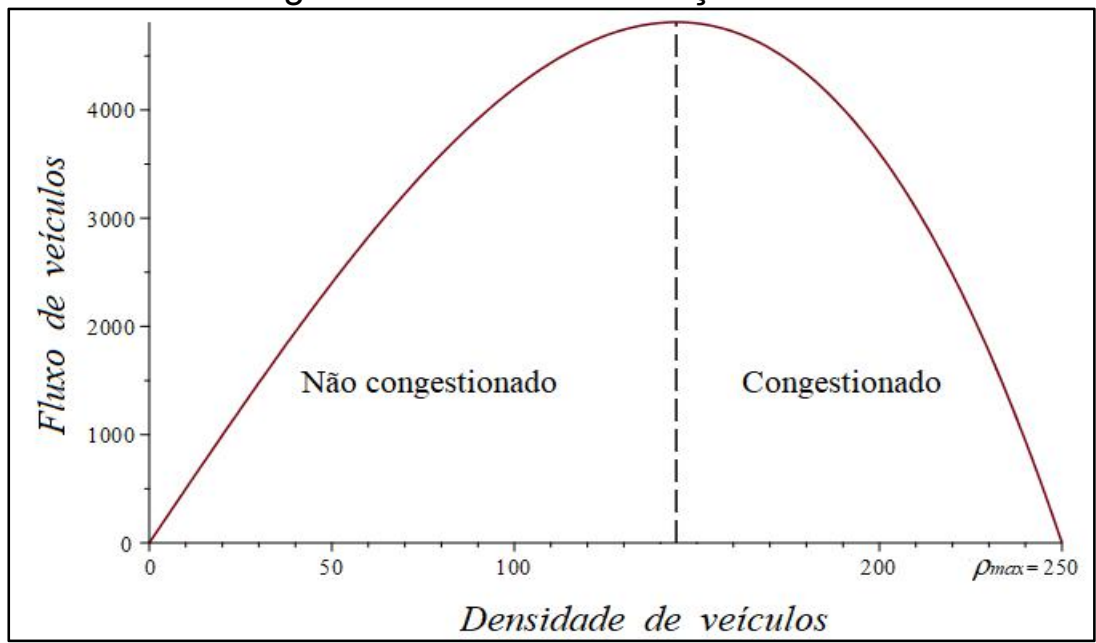

Fonte: Elaboração dos autores (2020).

- Se um sinal verde permanece aberto por dois minutos, $T=2 \mathrm{~min}$, um determinado veículo a uma distância $x_{0}=25 \mathrm{~m}$ à esquerda do sinal (em $\mathrm{x}=0$ ) levará um tempo igual a $1,299 \times 10^{-3} h$, isto é, aproximadamente 4,68 segundos para atravessá-lo com luz verde. Esse valor do tempo é obtido diretamente da equação (60). Usando a equação (61) esse carro passará no sinal aberto com a velocidade de aproximadamente $42,9 \mathrm{~km} / \mathrm{h}$.

- Supondo que o sinal em luz verde leva um tempo $T=2 \mathrm{~min}$ para fechar, então o número de carros que terá passado com o sinal aberto é dado por

(i) No caso linear:

$$
N_{L}=\rho_{\max }\left(\frac{u_{\max } T}{4}\right) \approx 104 \text { carros }
$$

onde $N_{L}=\rho_{\max } d_{\max }$ com distância máxima $d_{\max }=\frac{u_{\max } T}{4}$.

(ii) No caso quadrático

$$
N_{Q}=\rho_{\max }\left(\frac{2 \sqrt{3} u_{\max } T}{9}\right) \approx 160 \text { carros }
$$

onde $N_{Q}=\rho_{\max } d_{\max }$ com distância máxima $d_{\max }=\frac{2 \sqrt{3} u_{\max } T}{9}$.

A Figura 3 mostra carros parados em um sinal fechado e logo após a abertura do sinal. 
Figura 3 - (a) Carros parados no sinal; (b) Sinal aberto.
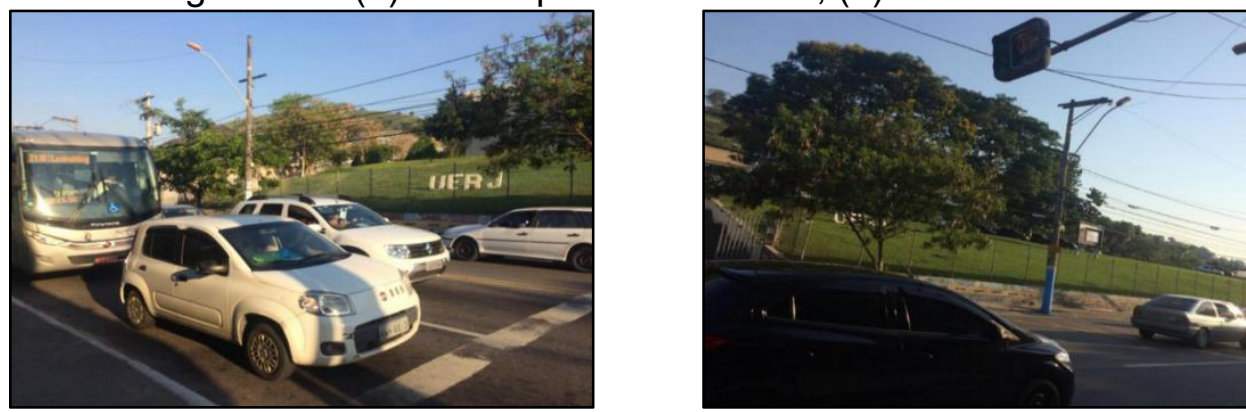

Fonte: Elaboração dos autores (2020).

Usando a equação (52), pode-se obter o gráfico da função de densidade de tráfego antes e após a abertura do sinal conforme mostra a Figura 4.

Figura 4 - Gráfico da Função densidade equação (51).

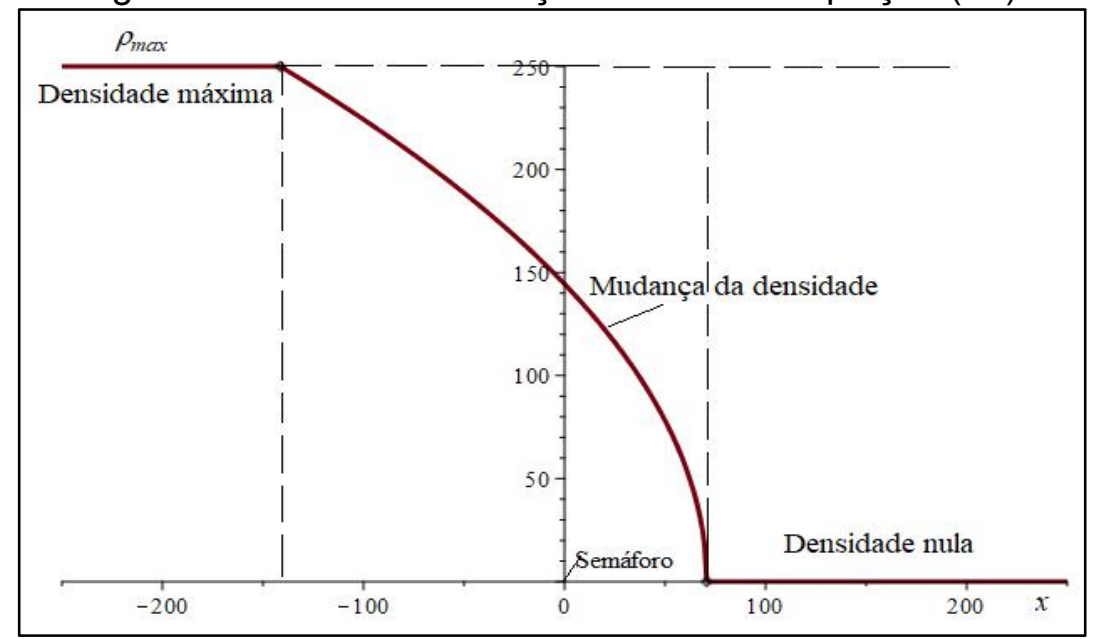

Fonte: Adaptado de Gómes Hernández (2009, p. 31).

A Figura 5 mostra um carro aproximando-se da velocidade máxima com o aumento do tempo.

Figura 5 - Trajetória de um veículo na rarefação.

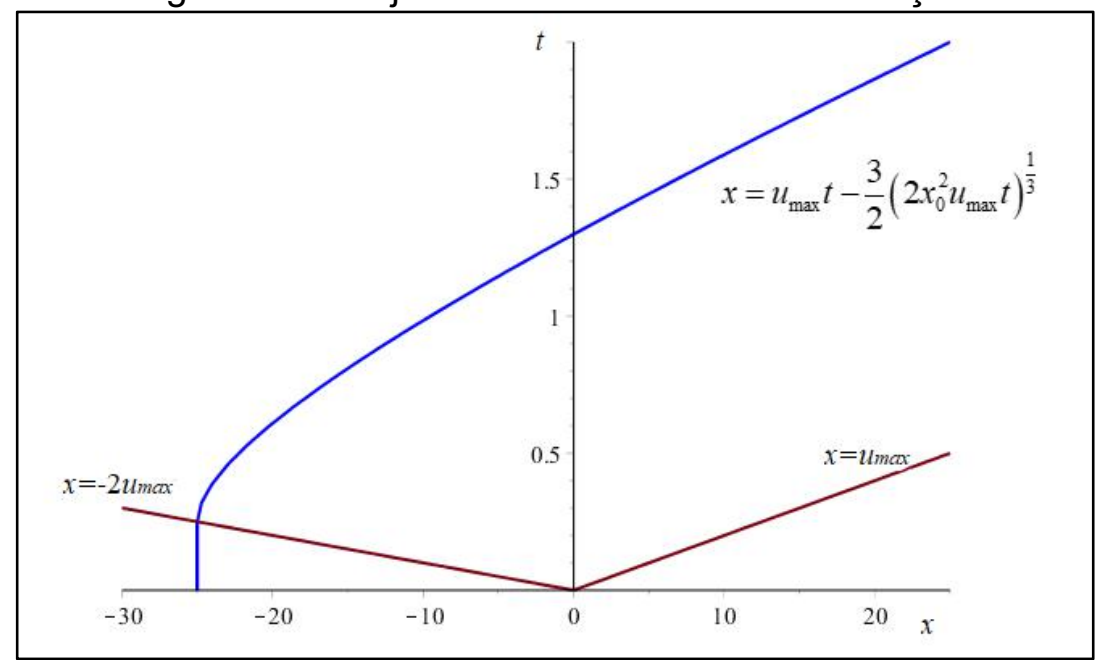

Fonte: Elaboração dos autores (2020). 


\section{Conclusões}

A solução entrópica para o problema de fluxo de tráfego em uma rodovia com conservação de fluxo de carros, considerando diferentes modelos para o campo de velocidade em função da densidade de veículos, foi obtida usando duas diferentes metodologias. Uma delas envolve a resolução de equação algébrica em função da densidade de tráfego e a outra utiliza a parametrização $x_{0}=x_{0}(x, t)$ com a regularização dos dados iniciais. A metodologia usando equações algébricas é mais restritiva, tendo sido adotada para uma região particular do plano em que as características partem da origem. Por outro lado, a parametrização $x_{0}=x_{0}(x, t)$ pode ser impossível de ser obtida explicitamente dependendo do modelo adotado. A simulação realizada com os modelos linear e quadrático mostrou que o tempo com que um motorista recebe a informação de que deve começar a acelerar, após a abertura no sinal com o modelo quadrático, é a metade do tempo de reação em relação ao modelo linear. Destaca-se ainda que o fluxo de veículos que passa pelo sinal aberto durante o tempo de exposição de luz verde é bem mais intenso com o modelo quadrático.

\section{Referências}

CUMINATO, J. A.; MENEGUETTE JUNIOR, M. Discretização de equações diferenciais parciais: técnicas de diferenças finitas. Rio de Janeiro: SBM, 2013.

FOX, R.W.; PRITCHARD, P. J.; MCDONALD, A. T. Introdução à mecânica dos fluidos. Trad. e revisão técnica: KOURY, Ricardo Koury; MACHADO, Luiz. 7. ed. Rio de Janeiro: LTC, 2015.

GÓMES HERNÁNDEZ, E. Desarrollo de un Modelo de Simulación Vehicular para la Mejora en la Sincronización de Semáforos. 2009. 127 f. Dissertação (Mestrado em Ciências da Computação) - Instituto Nacional de Astrofísica, Óptica y Electrónica, Tonantzintla, Puebla, México, 2009.

GUIDORIZZI, H. L. Um curso de cálculo. v. 3, 6. ed. Rio de Janeiro: LTC, 2019.

HABERMAN, R. Mathematical models: Mechanical vibrations, population dynamics and traffic flow. Philadelphia: SIAM, 1977.

LEVEQUE, R. J. Numerical methods for conservation laws. Lectures in Mathematics ETH Zurich. 2. ed. Basel: Birkhäuser, 1992.

WILLIAMSON, R. E.; CROWELL, R. H.; TROTTER, H. F. Cálculo de funções vetoriais. v. 2. Rio de Janeiro: LTC, 1979. 